\title{
Negative HER2/neu Amplification Using Immunohistochemistry and Chromogenic in Situ Hybridization Techniques in Skin Melanoma Cases
}

\author{
Nasrin Shayanfar ${ }^{1,2}$, Leila Bahari ${ }^{2}$, Zahra Safaie-Naraghi ${ }^{3}$, Kambiz Kamyab ${ }^{3}$, \\ Elmira Gheytanchi ${ }^{1}$, Nima Rezaei ${ }^{4,5}$
}

\begin{abstract}
Background: This study was performed to evaluate the amplification of HER-2/neu in patients with melanoma. Materials and Methods: Amplification of HER-2/neu was evaluated in a group of patients with melanoma, referred to two referral centers in Tehran, using immunohistochemistry (IHC) and chromogenic in situ hybridization (CISH) techniques. Results: Forty patients with mean age $57.9 \pm 19.5$ years were enrolled in this study. The most frequent type of melanoma was acral, while lower limbs were the most frequent sites. The amplification of HER2/ neu was negative in $97.5 \%$ of patients with IHC and in $100 \%$ of patients with CISH technique. Only one case $(2.5 \%)$ shows weak positive staining $(+2)$ in IHC method. Fifty five percent of melanoma was ulcerative, and the most common stages of tumors were stages $4 \mathrm{~b}$ and $3 \mathrm{~b}$. More than $47 \%$ of cases were in Clark level III, while the mean of Breslow thickness was $3.56 \pm 2.87 \mathrm{~mm}$. The stage of the case that showed weakly positive staining (2+) in IHC was 4b. Conclusions: The amplification of HER2/neu biomarker was negative in patients with melanoma, using both CISH and IHC techniques.
\end{abstract}

Keywords: Melanoma - HER2/neu - amplification - IHC - CISH

Asian Pac J Cancer Prev, 16 (2), 421-425

\section{Introduction}

Melanoma, the most serious type of skin cancer, develops in the melanocytes which can form in eyes and, rarely in internal organs, such as intestines (Jerant et al., 2000; Lens and Dawes, 2004; Parkin et al., 2005; Narin and Tuncay, 2012). The incidence and mortality rate of the melanoma have been increased during recent years (Lens and Dawes, 2004; Cihan et al., 2013). It has been estimated that approximately, fifty thousand melanoma related deaths occur worldwide yearly (Jerant et al., 2000; Parkin et al., 2005; Kanavy and Gerstenblith, 2011). It is more common in female, which is mainly presented in the legs that differ to male individuals where the back is the most common site of involvement (Jerant et al., 2000; Parkin et al., 2005; Kanavy and Gerstenblith, 2011).

The geographic pattern of disease indicates the principal cause is ultraviolet (UV) exposure; however, the exact cause of all melanomas is not clear (Kanavy and Gerstenblith, 2011). HER-2/neu proto-oncogene is initially isolated from a human mammary carcinoma and a salivary gland adenocarcinoma. HER-2/ neu oncogene encodes a $185 \mathrm{kDa}$ cell surface glycoprotein and shows intrinsic tyrosine kinase activity that regulates cell growth and differentiation (Ullrich and Schlessinger, 1990). HER-2 is a member of epidermal growth factor (EGF) receptor family and amplification or overexpression of this oncogene has been shown to play an important role in the development and progression of certain aggressive types of malignancy including ovarian, lung, gastric, endometrial and glioblastomas (Hetzel et al., 1992; Hiesiger et al., 1993; Tsugawa et al., 1993; Yu et al., 1994; Easty et al., 1995). The studies in this field are limited and the extent to which HER-2/neu is amplified in melanomas is not well known (Rongcun et al., 1999). During recent years, the HER-2/neu has become as an important biomarker and target of therapy for breast cancer. The verification of overexpression of HER-2/neu in patients with melanoma would be a remarkable scientific progress in understanding of melanogenesis and may feasibly lead to the diagnosis in early stage and effective treatment (Potti et al., 2004). The studies have confirmed HER2 expression with the HercepTestTM (DAKO) immunohistochemical (IHC) assay for evaluation of HER2 expression in breast cancer. Moreover these studies indicated that CISH is a useful alternative for detection of HER2 amplification in tumor samples, especially for confirming the IHC staining results (Tanner et al., 2000). Therefore, to shed light to the

${ }^{1}$ Oncopathology Research Center, ${ }^{2}$ Department of Pathology, Iran University of Medical Sciences, ${ }^{3}$ Department of Pathology, Razi Hospital, ${ }^{4}$ Research Center for Immunodeficiencies, Children's Medical Center, ${ }^{5}$ Molecular Immunology Research Center, Department of Immunology, School of Medicine, Tehran University of Medical Sciences, Tehran, Iran ${ }^{\star}$ Equal contributors ${ }^{*}$ For correspondence:nasrin.shayanfar@gmail.com 
over expression of HER-2/neu in patients with melanoma, this study was performed to evaluate the amplification of HER2/neu, using IHC and CISH techniques.

\section{Materials and Methods}

\section{Study design}

In this cross sectional study, 40 patients with melanoma who were referred to the Rasoul-e-Akram General Hospital and the Razi Dermatology Hospitals during 20062011 were recruited. The patients' information, including age, sex, tumor type, site, stage, thickness and depth of the tumor were recorded. Then the samples were evaluated to select the best section of the tumor for IHC and CISH. To evaluate the amplification of HER2/neu, 40 paraffin blocks were retrieved from archive of pathology wards and one cut (4-6 mm) for IHC and 2 cuts $(3 \mathrm{~mm}$ ) for CISH were selected from each block. IHC was performed in the Rasoul-e-Akram pathology laboratory (EnVision method) and CISH in the Oncopathology Research Center of Iran University of Medical Sciences.

\section{IHC method}

Immunohistochemical staining was performed on 4-5 $\mu \mathrm{m}$ thick FFPE tissue sections mounted on poly-l-lysine pre-coated slides using EnVision method (Gheytanchi et al., 2014). Sections were deparaffinized, using xylene and alcohol 100o and 96o and stained, using rabbit anti human HER2 polyclonal antibody (Code: A0485Dako, Copenhagen, Denmark). Heat-induced antigen retrieval was applied, using autoclave method and DAKO target retrieval solution. After washing with phosphate buffer saline (PBS), specimens were incubated with antibodies for $90 \mathrm{~min}$ at room temperature. Then, samples were incubated for $45 \mathrm{~min}$ with DAKO EnVision system at room temperature. Visualization of the reaction was acquired using diaminobenzidine (DAB) solution. Staining with Mayer's hematoxylin was performed afterwards. At each staining session, HER2 positive breast invasive ductal carcinoma was used as positive controls, and negative controls were prepared through elimination of antibody. Then the slides were observed by light microscope. The slides were scored based on ASCO/CAP criteria.

\section{IHC evaluation and scoring}

The immune-positivity for HER-2/neu was appeared as membranous reaction and sometimes cytoplasmic. The positivity of the immunostaining was determined in 10 different fields at $400 \mathrm{x}$ magnification and recorded as negative (score 0 and $1+$ ), weakly positive (score $2+$ ), and positive (score $3+$ ) in more than $30 \%$ of the tumor cells.

\section{CISH procedure}

CISH was performed based on ZytoDot 2C SPEC HER2/CEN 17 Probe kit (Mayr et al., 2009; Hwang et al., 2011; Gleeson et al., 2014), using in vitro diagnostic medical device (IVD) and CE Mark EV directive 98/79/ EC. The PD1 2 contains two polynucleotide probes. The presence of certain nucleic acid sequences in cells or tissue can be detected by in situ hybridization, using labeled DNA probes. The hybridization results in duplex formation of sequences present in the test object with the labeled DNA probe. Duplex formation of the labeled probe (with sequences of HER2 and chromosome 17 alpha-satellites in the test material) can be visualized using primary (unmarked) antibodies, which are detected by secondary polymerized enzyme-conjugated antibodies. The enzymatic reactions of chromogenic substrates lead to the formation of colored signals that can be visualized by light microscope. The polynucleotides contained in the ZytoDot 2C SPEC HER2/CEN 17Probe EmaNOF, which recognize the alpha-satellite-sequences of the centromere of chromosome 17 function in themselves as an internal control that a successful hybridization has occurred, as well as proving the integrity of the cellular DNA. Then the Anti-DIG/Anti-DNP-MIX (AB3) solution was added as initial antibody and then recognized by antibodies containing conjugated polymerase enzyme. Then colored solutions were added and red and green signals are detectable by light microscope at $\times 100$. These steps occurred during 2-4 days as follow:

\section{Step I. Preparatory step (first and second day)}

Step II. Pretreatment step including heating and incubation in xylene, ethanol, H2O2 (3\%) and EDTA

In this step the pretreatment solution (PT2) was heated, digested using pepsin Solution (ES1,3-10 minutes) and dehydrated using alcohol.

Step III. Hybridization (PD12) and incubation in 37oC for one night

Step IV: post hybridization including washing, final staining and observing under light microscope

Under light microscope at power 100 the signals of hybridization of copy of HER2 was seen as green spot signal while the signals of hybridization of chromosome 17 is seen as a red spot signal that it is differentiated from context (blue H,E).

The prepared slides were observed by two pathologists based on ASCO/CAP grading guideline.

The stained slides (IHC method) were interpreted using Hercep test as follow: Negative (Score 0 or Score $1+) *$ Score 0 : No staining is observed or membrane staining is observed in less than $10 \%$ of the tumor cells; *Score 1+: A faint/barely perceptible membrane stain is detected in more than $10 \%$ of the tumor cells; The cells are only stained in part of their membrane

Weakly positive (Score $2+$ ) *Score $2+$ : A weak to moderate complete membrane staining is observed in more than $10 \%$ of the tumor cells; Strongly positive (Score $3+$ ); ${ }^{*}$ Score $3+:$ A strong complete membrane staining is observed in more than $10 \%$ of the tumor cells

The stained slides (CISH method) were interpreted using ASCO/CAP as follow:

Under light microscope at $1000 \times$, HER 2 signals in nuclei were counted and the amplification gene was identified. If spots were lower than 6 , the gene was considered unamplified and amplification was defined when the spots $\geq 6$ in more than $50 \%$ of cancer cells or a small cluster copy of gene (amplified) was present.

If the sample was homogenous at least 30 cancer 
cells from each sample were recruited but if the sample contains 5-10 copy of HER2 gene in less than 50\% of selected area, at least 60 cancer cells from each sample were evaluated. Furthermore, the number of cancer cells and ability to detect of the normal copy of HER 2 gene ratio was measured.

The Zytovision test has green probe for HER2 gene and red probe for chromosome 17. These probes were recorded in invasive tumor in area that at least had 20 cells; also uncounted areas were less than $25 \%$ and were calculated as follow:

HER2/CEP17 <1.8= negative

HER2/CEP17 1.8-2.2= equivocal

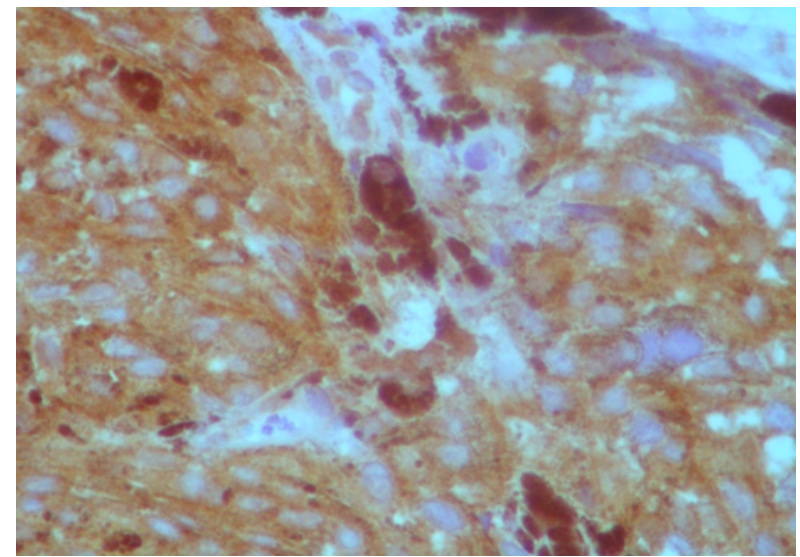

Figure 1. Negative Immunoreactivity for HER2/neu (Immunohistochemistry), Magnification (40x)

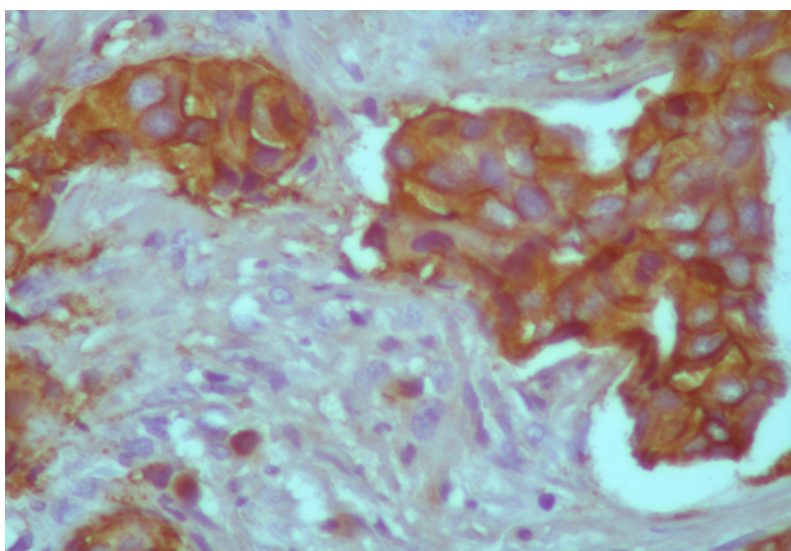

Figure 2. Weak Positive (2+) Staining of one case by Immunohistochemistry, Magnification (20x)

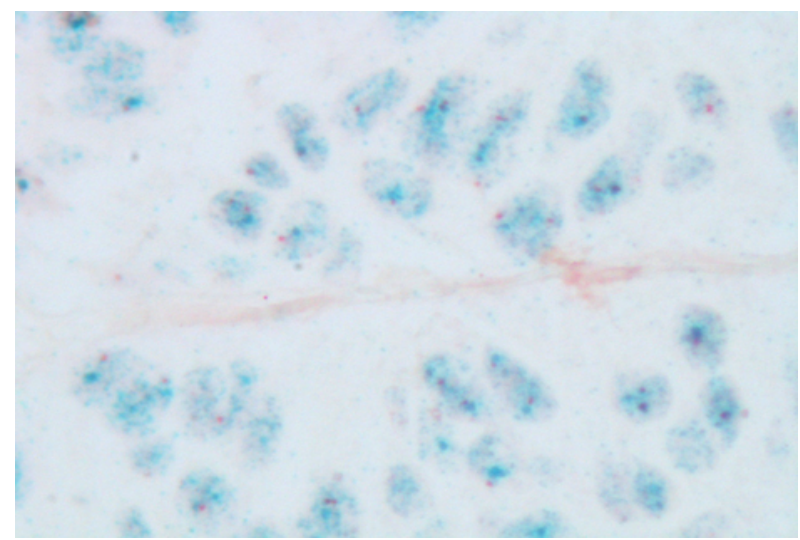

Figure 3. No Amplication of HER2/neu by Chromogenic in Situ Hybridization (CISH), Magnification (100x)
HER $2 / C E P 17>2.2=$ positive

\section{Statistical analysis}

Data were analyzed using Statistical Package for Social Studies version 21 (SPSS Inc, Chicago, Ill). Categorical data are presented as numbers (\%), and continuous data as mean $\pm \mathrm{SD}$. We used Chi square test to compare categorical variables. P value of less than 0.05 was considered significant.

\section{Results}

Forty patients, including 19 male $(47.5 \%)$ and 21 female $(52.5 \%)$, with mean age of $57.9 \pm 19.51$ (range: 10-89) years were evaluated. The most common type of melanoma was acral type, followed by nodular and lentigo maligna melanoma, whereas the most common site was lower limb that in 22 patients $(55 \%)$ was ulcerative (Table 1). Majority of tumors were in stage $4 b(25 \%)$, followed by $1 \mathrm{a}(20 \%)$. Regarding amplification of HER2/neu in IHC technique, 39 patients $(97.5 \%)$ showed negative (Figure 1) amplification (score 0 and 1+), while one patient was weekly positive (score 2+, Figure 2); however, in CISH technique, the amplification in all samples were negative (Table 1, Figure 3). The stage of one tumor that showed

Table 1. Clinicopathological Characteristics of Melanoma Patients

\begin{tabular}{|c|c|c|}
\hline \multirow[t]{5}{*}{ Age } & $<20$ & $1(2.5 \%)$ \\
\hline & $20-39$ & $5(12.5 \%)$ \\
\hline & $40-59$ & $12(30 \%)$ \\
\hline & $60-80$ & $18(45 \%)$ \\
\hline & $>80$ & $4(10 \%)$ \\
\hline \multirow[t]{5}{*}{ Melanoma subtypes } & Acral & $21(52.5 \%)$ \\
\hline & nodular & $7(17.5 \%)$ \\
\hline & lentigo & $7(17.5 \%)$ \\
\hline & superficial & $3(7.5 \%)$ \\
\hline & nevoid & $2(5 \%)$ \\
\hline \multirow[t]{4}{*}{ Location } & Lower limb & $22(55 \%)$ \\
\hline & Head and neck & $11(27.5 \%)$ \\
\hline & trunk & $4(10 \%)$ \\
\hline & Upper limb & $3(7.5 \%)$ \\
\hline Ulcer & Presence & $22(55 \%)$ \\
\hline \multirow[t]{8}{*}{ Tumor staging } & $1 \mathrm{a}$ & $8(20 \%)$ \\
\hline & $1 b$ & $1(2.5 \%)$ \\
\hline & $2 \mathrm{a}$ & $1(2.5 \%)$ \\
\hline & $2 b$ & $1(2.5 \%)$ \\
\hline & $3 a$ & $5(12.5 \%)$ \\
\hline & $3 b$ & $9(22.5 \%)$ \\
\hline & $4 a$ & $4(10 \%)$ \\
\hline & $4 b$ & $10(25 \%)$ \\
\hline \multirow[t]{3}{*}{$\mathrm{IHC}$} & Score 0 & $30(75 \%)$ \\
\hline & Score $1+$ & $9(22.5 \%)$ \\
\hline & Score $2+$ & $1(2.5 \%)$ \\
\hline \multirow[t]{5}{*}{ Clark level } & I & $1(2.5 \%)$ \\
\hline & II & $5(125 \%)$ \\
\hline & III & $20(50 \%)$ \\
\hline & IV & $8(20 \%)$ \\
\hline & $\mathrm{V}$ & $5(7.5 \%)$ \\
\hline \multirow[t]{4}{*}{ Breslow Thickness (mm) } & $<0.7$ & $4(10 \%)$ \\
\hline & $0.8-1.6$ & $6(15 \%)$ \\
\hline & $1.7-3.6$ & $13(32.5 \%)$ \\
\hline & $>3.6$ & $16(40 \%)$ \\
\hline
\end{tabular}


Nasrin Shayanfar et al

overexpression in IHC was 4b. Mean of Breslow thickness was $3.56 \pm 2.87$ (range: $0.5-15 \mathrm{~mm}$ ); the most common Clark level of sample was III (Table 1).

\section{Discussion}

Overexpression of the HER2 protein generally results from HER2 gene amplification (Pauletti et al., 1996). Some studies signified HER-2/neu as a prognostic predictive factor in several cancers, such as breast cancer, ovarian, non-small cell lung, and gastric cancer (Gershenson et al., 1996; Allgayer et al., 2000; Bar-Sela et al., 2003). In this study, we evaluated HER $2 /$ neu gene amplification in 40 patients with cutaneous melanoma and obtained similar results with two techniques IHC and CISH (97.5\% and 100\% negative, respectively). Only one patient in IHC technique showed weakly positive immune staining (2+); however, in CISH technique, the amplification in all samples was negative. In other word, $97.5 \%$ of samples were negative in this study that was in line with Potti et al study (Potti et al., 2003) that signified $0.9 \%$ of melanoma showed HER-2/neu overexpression by immunohistochemistry. Another trial in agreement with these findings conducted by El-Shaikh et al that showed negative HER2/neu overexpression in more than $90 \%$ of their patients (El-Sheikh S, 2009). Furthermore, another experience in line with our findings conducted by Easty et al indicated variable levels of HER-2/neu mRNA in 19 melanoma cell lines (Easty et al., 1995). Moreover, in a study in 16 additional melanoma cell lines, ChenevixTrench et al signified low level of HER-2/neu mRNA (Chenevix-Trench et al., 1990). However, in contrast to our findings and mentioned studies above, Persons et al signified that HER-2/neu is amplified in up to $13.16 \%$ of melanomas (Persons et al., 2000). Bodey et al applied immunohistochemistry technique and reported that HER2/neu expressed in $40 \%$ of primary cutaneous melanoma (Bodey et al., 1997). The reason for such discrepancy is not clear, but it may be related to difference in patients' selection, study design and staining technique and different interpretation of the slides. On the other hand, some studies revealed HER2 overexpression in normal skin tissue and non-melanoma cancer (Krahn et al., 2001). Therefore, HER-2/neu overexpression is not specific for melanoma and it appears to have no value as a biomarker in patients with skin melanoma.

In this study, the frequency of melanoma in female was slightly more than male and the mean age was 57 years. Conversely, the results observed by Demierre et al mentioned melanoma is more common in males, but in agreement with findings of this study, it is more common after fifty (Demierre et al., 2005). El-Sheikh et al in another study reported similar results with Demierre et al in age and sex distribution (El-Sheikh, 2009).

The most common type of melanoma in this work was acral followed by nodular and lentigo maligna type that was in line with Demierre et al study that found nodular melanoma is the second most common type of cutaneous melanoma; however, in their survey, the most common type was superficial spreading type (Demierre et al., 2005). In the El-Sheikh et al study (2009), the type of melanoma in all patients was nodular.

The most common site of melanoma in present study was lower limb, followed by head and neck, conversely in the Elshaikh et al study the most common site was face and forearm, regarding this fact some studies declare the site of the melanoma may be related to prognosis (Levi et al., 1998), but is not consistent with other experiences that have not shown any correlation (Hoersch et al., 2006).

The most of samples in current survey were in Clark level III moreover Breslow thickness in most of the samples was more than $3.6 \mathrm{~mm}$. Conversely, in a study by Safaie on 47 patients with melanoma, more than $42 \%$ of cases were in Clark level I, and in $53 \%$ of patients, Breslow thickness was equal to or less than 0.75 millimeter (Safaie-Naraghi et al., 2006). The melanoma in 55\% of our patients was ulcerative and the most of tumors were in stage $4 \mathrm{~b}(25 \%)$. Moreover the stage of one tumor that showed overexpression in IHC was $4 \mathrm{~b}$.

It could be suggested that the amplification of HER2/ neu biomarker was negative in patients with melanoma, using both CISH and IHC techniques. However, any conclusion from this study is limited by low number of enrolled cases, which is consistent with previous studies in this field as well.

\section{Acknowledgements}

We would like to thank the staff of the Department of Pathology in the Rasoul-e-Akram and the Razi hospitals for their contribution to the maintenance of our patient record without which this project would have been impossible.

\section{References}

Allgayer H, R. Babic, et al (2000). c-erbB-2 is of independent prognostic relevance in gastric cancer and is associated with the expression of tumor-associated protease systems. J Clin Oncol, 18, 2201-9.

Bar-Sela G, Kuten A, Ben-Eliezer S, Gov-Ari E, Ben-Izhak O (2003). Expression of HER2 and C-KIT in nasopharyngeal carcinoma: implications for a new therapeutic approach. Mod Pathol, 16, 1035-40.

Bodey B, Bodey Jr B, Groger AM, et al (1997). Clinical and prognostic significance of the expression of the c-erbB-2 and c-erbB-3 oncoproteins in primary and metastatic malignant melanomas and breast carcinomas. Anticancer Res, 17, 1319-30.

Chenevix-Trench G, Martin NG, Ellem KA et al (1990). Gene expression in melanoma cell lines and cultured melanocytes: correlation between levels of c-src-1, c-myc and p53. Oncogene, 5, 1187-93.

Cihan YB, Baykan H, Kavuncuoglu E, et al (2013). Relationships between skin cancers and blood groups--link between nonmelanomas and $\mathrm{ABO} / \mathrm{Rh}$ factors. Asian Pac J Cancer Prev, 14, 4199-4203.

Demierre MF, Chung C, Miller DR, Geller AC (2005). Early detection of thick melanomas in the United States: beware of the nodular subtype. Arch Dermatol, 141, 745-50.

Easty DJ, Herlyn M, Bennett DC (1995). Abnormal protein tyrosine kinase gene expression during melanoma progression and metastasis. Int J Cancer, 60, 129-36.

El-Sheikh S, El-Sheikh S, El-Morsy I (2009). Detection of c-Kit 
(CD117) and HER-2/neu in Oral and Cutaneous Malignant Melanomas. J Egypt Women DermatolSoc, 6, 66-73.

Gershenson DM, Tortolero-Luna G, Malpica A, et al (1996). Ovarian intraepithelial neoplasia and ovarian cancer. Obstet Gynecol Clin North Am, 23, 475-543.

Gheytanchi E, Mehrazma M, Madjd M (2014). Expression of $\mathrm{Ki}-67, \mathrm{p} 53$ and VEGF in pediatric neuroblastoma. Asian Pac J Cancer Prev, 15, 3065-70.

Gleeson G, Larkin A, Horgan N, Kennedy S (2014). Evaluation of chromogenic in situ hybridization for the determination of monosomy 3 in uveal melanoma. Arch Pathol Lab Med, 138, 664-70.

Hetzel DJ, Wilson TO, Keeney GL, et al (1992). HER-2/neu expression: a major prognostic factor in endometrial cancer. Gynecol Oncol, 47, 179-85.

Hiesiger EM, Hayes RL, Pierz DM, Budzilovich GN (1993). Prognostic relevance of epidermal growth factor receptor (EGF-R) and c-neu/erbB2 expression in glioblastomas (GBMs). J Neurooncol, 16, 93-104.

Hoersch B, Leiter U, Garbe C (2006). Is head and neck melanoma a distinct entity? A clinical registry-based comparative study in 5702 patients with melanoma. Br J Dermatol, 155, 771-7.

Hwang CC, Pintye M,Chang LC, et al (2011). Dual-colour chromogenic in-situ hybridization is a potential alternative to fluorescence in-situ hybridization in HER2 testing. Histopathology, 59, 984-92.

Jerant AF, Johnson JT, Sheridan CD, Caffrey TJ (2000). Early detection and treatment of skin cancer. Am Fam Physician, 62, 357-68.

Kanavy HE, Gerstenblith MR (2011). Ultraviolet radiation and melanoma. Semin Cutan Med Surg, 30, 222-8.

Krahn G,Leiter U,Kaskel P, et al (2001). Coexpression patterns of EGFR, HER2, HER3 and HER4 in non-melanoma skin cancer. Eur J Cancer, 37, 251-9.

Lens MB, Dawes M (2004). Global perspectives of contemporary epidemiological trends of cutaneous malignant melanoma. Br J Dermatol, 150, 179-85.

Levi F, Randimbison L, La Vecchia C, Te VC, Franceschi S (1998). Prognostic factors for cutaneous malignant melanoma in Vaud, Switzerland. Int J Cancer, 78, 315-19.

Mayr D, Heim S, Weyrauch K, et al (2009). Chromogenic in situ hybridization for Her-2/neu-oncogene in breast cancer: comparison of a new dual-colour chromogenic in situ hybridization with immunohistochemistry and fluorescence in situ hybridization. Histopathology, 55, 716-23.

Narin A, Tuncay O (2012). Relationships between malignant melanoma and chromosome damage in human peripheral blood lymphocytes. Asian Pac J Cancer Prev, 13, 5229-32.

Parkin DM, Bray F, Ferlay J, Pisani P (2005). Global cancer statistics, 2002. CA Cancer J Clin, 55, 74-108.

Pauletti G, Godolphin W, Press MF, Slamon DJ (1996). Detection and quantitation of HER-2/neu gene amplification in human breast cancer archival material using fluorescence in situ hybridization. Oncogene, 13, 63-72.

Persons DL, Arber DA, Sosman JA, Borelli KA, Slovak ML (2000). Amplification and overexpression of HER-2/neu are uncommon in advanced stage melanoma. Anticancer Res, 20, 1965-8.

Potti A, Hille R, Koch M (2003). Immunohistochemical determination of HER-2/neu in malignant melanoma. Anticancer Res, 23, 4067-9.

Potti A, Moazzam N, Langness E, et al (2004). Immunohistochemical determination of HER-2/neu, c-Kit (CD117), and vascular endothelial growth factor (VEGF) overexpression in malignant melanoma. J Cancer Res Clin Oncol, 130, 80-6.

Rongcun Y, Salazar-Onfray F, Charo J, et al (1999). Identification of new HER2/neu-derived peptide epitopes that can elicit specific CTL against autologous and allogeneic carcinomas and melanomas. J Immunol, 163, 1037-44.

Safaie-Naraghi Z, Bahadori M, Ehsani AH, et al (2006). Evaluation of primary cutaneous malignant melanoma according to Breslow and Clarke pathological indices. Tehran University Medical Journal, 64, 79-85.

Tanner M, Gancberg D, Di Leo A, et al (2000). Chromogenic in situ hybridization: a practical alternative for fluorescence in situ hybridization to detect HER-2/neu oncogene amplification in archival breast cancer samples. Am J Pathol, 157, 1467-72.

Tsugawa K, Fushida S, Yonemura Y (1993). Amplification of the c-erbB-2 gene in gastric carcinoma: correlation with survival. Oncology, 50, 418-25.

Ullrich A, Schlessinger J (1990). Signal transduction by receptors with tyrosine kinase activity. Cell, 61, 203-12.

Yu D, Wang SS, Dulski KM, et al (1994). c-erbB-2/neu overexpression enhances metastatic potential of human lung cancer cells by induction of metastasis-associated properties. Cancer Res, 54, 3260-6. 